\title{
Novel TSC1 Gene Mutation in a Familial Case of Tuberous Sclerosis Complex: A Case Report
}

Alberto Pinzon-Ardila ${ }^{1,2 *}$, Claudia Vallin², Kevin Abrams ${ }^{1}$ and lan Miller $^{3}$

${ }^{1}$ Neuroscience Center, Baptist Health South Florida, 8900 N Kendall Dr, Miami, FL, USA

${ }^{2}$ The Neurology Group. 9090 SW 87 Ct. Suite 200, Miami, FL, USA

${ }^{3}$ Brain Institute, Nicklaus Children's Hospital. 3100 SW 62nd Ave. Miami, FL, USA

\begin{abstract}
Introduction: Tuberous sclerosis complex (TSC) is a genetic disorder that can arise from sporadic or inherited mutations in TSC1 or TSC2 genes. It is characterized by tumors in multiple organs that result in broad clinical manifestations, usually affecting the central nervous system. Diagnosis is based upon clinical criteria that require careful clinical examination along with imaging and the availability of genetic testing to assess gene mutations linked to this disorder. We present a familial case of tuberous sclerosis complex with multiple subtle clinical manifestations and novel TSC1 mutation that were diagnosed in adulthood. This report adds to the growing literature on TSC1 gene by correlating specific nucleotide substitutions with possible secondary clinical manifestations.

Case presentation: A 21 year-old Hispanic female presented with history of epilepsy onset in early childhood. Upon clinical examination and imaging testing, she was found to have lesions on her scalp, eyes and brain. Her mother is 51 years old with long-standing history of epilepsy with multiple lesions on skin, eyes, and similar findings in brain imaging to daughter. No additional family history was obtained suggestive of tuberous sclerosis complex.

Conclusion: Milder forms of tuberous sclerosis complex can remain undiagnosed until adolescence and adulthood, which may lead to treatment delay and complications due to the lack of regular follow-up care. In this case report, both individuals' presenting symptom was epilepsy and later other clinical findings were linked to the disorder. Additionally, a novel DNA sequence variant was detected, which expands our knowledge of known TSC1 gene mutations. Although these genetic test results cannot be used alone to make a definite diagnosis, they suggest an association to TSC given the clinical manifestations and family history.
\end{abstract}

Keywords: Epilepsy; Tuberous sclerosis complex; TSC novel mutation

\section{Introduction}

Tuberous sclerosis complex (TSC) is an autosomal dominant disorder that affects about 1:6,000 individuals. About two-thirds of TSC cases are sporadic, representing spontaneous mutations, while one-third are inherited [1]. It is characterized by the development of distinctive tumors termed hamartomas in multiple organ systems such as brain, retina, skin, heart, lungs, and kidneys [2]. TSC has been associated with mutations in either the TSC1 on chromosome 16p13.3 or TSC2 on chromosome 9q34, which encode hamartin and tuberin, respectively. The hamartin/tuberin complex formation is responsible for the signaling pathway that regulates a key inhibitor of cell growth and metabolism known as mammalian target of rapamycin complex 1 (mTORC1) [3].

TSC has high variability in clinical presentation and findings. Diagnosis of TSC is based on the presence of at least two major clinical features, or one major and two minor clinical features [4]. Although patients may present with early manifestations of seizures, intellectual disability, and skin abnormalities, fewer than $30 \%$ have all three and $6 \%$ have none [2]. Previous studies have revealed that patients with subtle TSC findings remain either without diagnosis or do not present symptoms until later in life (i.e., TSC identified in a parent after an affected child was diagnosed).

We are reporting a familial case of TSC with a novel TSC1 mutation in both affected individuals presenting with mild clinical manifestations.

\section{Case Presentation}

A 21-year-old Hispanic female presented with history of epilepsy, with seizure onset at age 6 . Her seizures are well controlled with medication. Her physical examination revealed a fibrous cephalic plaque $(3.5 \mathrm{~cm})$ and neurological examination was normal. On ophthalmic examination, small hamartomatous lesions about 200 to 300 microns were found. Baseline EEG showed right fronto-temporal intermittent discharge activity. A non-contrast brain CT depicted a single subependymal nodule (Figure 1A) and brain MRI demonstrated a subcortical tuber with associated radial migration line (Figure 1B). Renal ultrasound and echocardiogram results were normal and no overt pulmonary signs or symptoms (Figure 1C).

Her mother is 51 years old with long-standing history of epilepsy controlled with medication. On dermatological examination, there were inflamed pedunculated papules on the neck, trunk, and axillae consistent with soft fibromas. Multiple cherry angiomas and benign nevi were present on the trunk in addition to a hypomelanotic macule in the axilla (Figure 1F). Ophthalmic examination showed a few small

*Corresponding author: Alberto Pinzon-Ardila, The Neurology Group 9090 SW 87 Ct. Suite 200, Miami, FL, USA, Tel: 305.596.2080; Fax: 305.596.0657; E-mail: apneurobio@hotmail.com

Received September 29, 2015; Accepted November 30, 2015; Published December 10, 2015

Citation: Pinzon-Ardila A, Vallin C, Abrams K, Miller I (2015) Novel TSC1 Gene Mutation in a Familial Case of Tuberous Sclerosis Complex: A Case Report. J Epilepsy 1: 102. doi:10.4172/2472-0895.1000102

Copyright: $\odot 2015$ Pinzon-Ardila A, et al. This is an open-access article distributed under the terms of the Creative Commons Attribution License, which permits unrestricted use, distribution, and reproduction in any medium, provided the original author and source are credited. 
lesions consistent with retinal hamartomas. Brain MRI demonstrated a single subependymal nodule, subcortical tubers (Figure 1D), and radiation migration lines. Renal ultrasound, echocardiogram, and EEG results were normal and no overt pulmonary signs or symptoms (Figure 1E).

Genetic testing was conducted by Athena Diagnostics, Inc. TSC1 and TSC2 genes were analyzed for deletions by using Multiplex Ligation-mediated Probe Amplification (MLPA) of genomic DNA. DNA sequence analysis was performed by PCR amplification of purified genomic DNA, followed by automated DNA sequencing of the coding region and the 10 bases of intronic DNA surrounding each exon containing the highly conserved exon-intron splice junctions. This test detected a DNA sequence variant TSC1 IVS18-6 G>A that might interfere with normal splicing in both mother and daughter.

\section{Discussion}

TSC is characterized by multisystem involvement that results in a wide range of manifestations. Neurologic symptoms are common in individuals diagnosed with TSC, as epilepsy occurs in $80-90 \%$, cognitive impairment in $50 \%$, and neurobehavioral disorders in $>60 \%$ of cases [5]. However, milder forms of the disorder may lead to diagnosis during adolescence and adulthood when more evident manifestations are present.

We describe a mother and daughter with no overt cognitive changes and no other family history (neither had siblings) suggestive of TSC. The presenting symptom in both individuals was epilepsy and other clinical manifestations were later identified as part of TSC. They both fulfill definite diagnostic criteria as per the most recent diagnostic criteria [4]. The mother has subependymal nodules and cortical dysplasia as major features in addition to suspected retinal hamartomas and some dermatological findings, and the daughter has subependymal nodules, cortical dysplasia, fibrous plaque on scalp, and small retinal hamartomas.

Previous studies have reported that approximately $10-30 \%$ of all TSC cases are caused by TSC1 mutations [6]. TSC1 mutations are
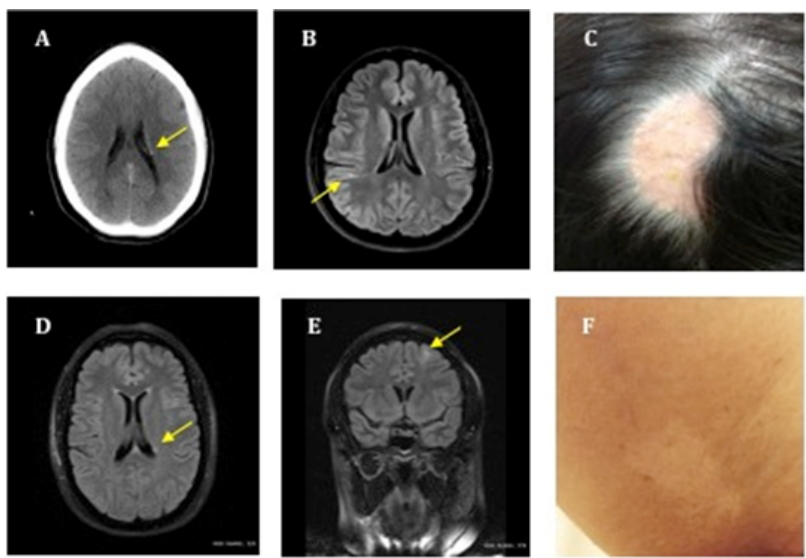

Figure 1: Daughter and mother's brain imaging and dermatological findings. (A) Non-contrast brain CT of daughter: single calcified subependymal nodule along the body left lateral ventricle. (B) Brain MRI-Axial FLAIR image of daughter: small right parietal subcortical tuber with associated radial migration line extending to the right lateral ventricle. (C) $3.5 \mathrm{~cm}$ fibrous cephalic plaque noted on daughter. (D) Brain MRI-Axial FLAIR image of mother: single subependymal nodule along the body of the left lateral ventricle. (E) Brain MRI-Coronal FLAIR image of mother: subcortical tuber along the left middle frontal gyrus. (F) Single hypomelanotic macule in the axilla on mother. associated with a less severe phenotype as compared to TSC2 gene [7]. Genetic testing in mother and daughter indicated a single nucleotide substitution of A-to-G that is likely pathogenic in TSC1 gene not previously described in the literature. These findings along with the clinical manifestations suggest this is a novel TSC-causing mutation.

\section{Conclusion}

This report describes a novel TSC1 mutation found in a mother and daughter with history of epilepsy and clinical criteria of TSC. Although it is not certain that the genetic test findings correlate with TSC, these patients' clinical manifestations and family history are suggestive of its association. This novel DNA sequence variant expands the spectrum of known TSC1 gene mutations. It supports the growing literature that familial cases with TSC1 mutations might have milder clinical manifestations. Misdiagnosis of these individuals can be avoided with careful clinical examination and genetic testing to be able to provide prompt treatment and management.

\section{Consent}

Written informed consent was obtained from the patients for publication of this case report and accompanying images.

\section{Competing Interests}

The authors declare that they have no competing interests.

\section{Author's Contributions}

APA developed manuscript concept, design, and performed draft revisions. CV performed manuscript draft and revisions. KA analyzed and interpreted imaging analyses and performed draft revisions. IA performed draft revision. All authors read and approved the final manuscript.

\section{Study Funding}

No targeted funding reported.

\section{References}

1. Osborne JP, Fryer A, Webb D (1991) Epidemiology of tuberous sclerosis. Annals of the New York Academy of Sciences 615: 125-127.

2. Gomez MR (1991) Phenotypes of the tuberous sclerosis complex with a revision of diagnostic criteria. Ann N Y Acad Sci 615: 1-7.

3. Napolioni V, Curatolo P (2008) Genetics and molecular biology of tuberous sclerosis complex. Curr. Genomics 9: 475-487.

4. Krueger DA, Northrup H (2013) Tuberous sclerosis complex surveillance and management: recommendations of the 2012 international tuberous sclerosis complex consensus conference. Pediatric Neurology 49: 255-265.

5. Thiele EA (2010) Managing and understanding epilepsy in tuberous sclerosis complex. Epilepsia 51: 90-91.

6. van Slegtenhorst M, de Hoogt R, Hermans C, Nellist M, Janssen B, et al. (1997) Identification of the tuberous sclerosis gene TSC1 on chromosome 9q34. Science 277: 805-808.

7. Au KS, Williams AT, Roach ES (2007) Genotype/phenotype correlation in 325 individuals referred for a diagnosis of tuberous sclerosis complex in the United States. Genet Med 9: 88-100. 